\title{
The proper motions of fundamental stars
}

\section{1535 stars from the Basic FK5^}

\author{
G. A. Gontcharov ${ }^{1}$, A. A. Andronova ${ }^{1}$, O. A. Titov ${ }^{2}$, and E. V. Kornilov ${ }^{1}$ \\ 1 Pulkovo Observatory, Saint-Petersburg 196140, Russia \\ e-mail: gaoran@mail. wplus.net \\ 2 Astronomical Institute, Saint-Petersburg State University, Saint-Petersburg, Russia \\ e-mail: olegtitov@mail.ru
}

Received 26 April 2000 / Accepted 28 September 2000

\begin{abstract}
A direct combination of the positions given in the HIPPARCOS catalogue with astrometric groundbased catalogues having epochs later than 1939 allows us to obtain new proper motions for the 1535 stars of the Basic FK5. The results are presented as the catalogue Proper Motions of Fundamental Stars (PMFS), Part I. The median precision of the proper motions is 0.5 mas/year for $\mu_{\alpha} \cos \delta$ and 0.7 mas/year for $\mu_{\delta}$. The non-linear motions of the photocentres of a few hundred astrometric binaries are separated into their linear and elliptic motions. Since the PMFS proper motions do not include the information given by the proper motions from other catalogues (HIPPARCOS, FK5, FK6, etc.) this catalogue can be used as an independent source of the proper motions of the fundamental stars.
\end{abstract}

Key words. astrometry - reference systems - catalogs

\section{Introduction}

The proper motions in the HIPPARCOS catalogue (hereafter HIP) (ESA 1997) can be improved by combining the HIP and ground-based data. The reasons why this should be done are well-known. They are discussed, for example, in the description of recently realized FK6 catalogue, Part I, by Wielen et al. (1999). The FK6 is a combination of the HIP with FK5 (Fricke et al. 1988; Fricke et al. 1991). Part I contains 878 stars from the FK5 catalogue with little or no indication of duplicity. The FK6 shows nonlinear motions of some of the stars, but it cannot provide details of such motions because the ground-based data are represented by the compilation catalogue FK5.

To investigate the proper motions with emphasis on non-linear motions of stars the direct combinations of the HIP with ground-based catalogues were proposed by Gontcharov \& Kornilov (1997) as well as by Wielen et al. (1999) (see p. 6, 7) in their project "FK7". In the process the systematic errors in each ground-based catalogue have to be eliminated individually reducing of each catalogue to the HIPPARCOS system.

Send offprint requests to: G. A. Gontcharov

* Catalogue (Table 3 ) is only available at the CDS via anonymous ftp to cdsarc.u-strasbg.fr $(130.79 .128 .5)$ or via http://cdsweb.u-strastg.fr/cgi-bin/qcat?J/A+A/365/222
An advantage of this approach is that one can use the same procedure both for single and non-single, linearly and non-linearly moving stars. Therefore, proper motions of thousands of stars can be improved simultaneously. It would be reasonable to begin with 4638 stars common to the HIP and FK5 because they have been the most extensively observed from the ground. The Proper Motions of Fundamental Stars (PMFS) catalogue, Part I - 1535 stars from the Basic FK5 - is the result of our direct combination of the HIP with over 60 compiled and observational ground-based catalogues. The proper motions of the stars from the FK5 Extension will be considered in Part II of the PMFS.

\section{Selection of ground-based catalogues}

To trace the non-linear motions of the stars one should use the most precise observational catalogues with a spread in epoch, especially earlier ones. The catalogues should be evenly and closely (ideally annually) distributed over the time interval. Moreover, for correct estimation of the systematic errors every catalogue should contain reasonable number of stars and cover a rather wide interval of $\alpha$ and $\delta$. For the last reason we rejected several zonal catalogues, some obtained with astrolabes and some others.

We have analyzed a hundred observational catalogues as described in Gontcharov \& Andronova (1997), 
Andronova (1998) and Gontcharov et al. (1998). Hereafter we reject the observations made at zenith distances $>70^{\circ}$ as not being accurate enough. For the evaluation of the catalogues the mean absolute values of the differences $\Delta \alpha$ and $\Delta \delta$ in the sense "observational catalogue minus HIP at the observational catalogue epoch" were calculated twice for every catalogue: before and after the elimination of systematic errors as described in the section "Method". The procedure for the determination of the systematic errors was much less effective for older catalogues probably because of the degradation of the HIP positions at earlier epochs. In other words, the method of direct combination of the HIP with ground-based catalogues seems to be acceptable only for those catalogues with epochs at which the HIP (as the reference) is still several times more precise. It turns out that most catalogues observed after 1940 (as against a few observed before 1940) have mean absolute values of coordinate differences "catalogue minus HIP" < 150 mas after elimination of systematic errors.

In order to get a uniform and dense set of observational catalogues, we decided to reject all catalogues observed before 1939 as well as those with mean absolute value of coordinate differences "catalogue minus HIP" $<150$ mas after elimination of systematic errors. The final list of catalogues used for positions includes 57 observational catalogues with epochs between 1939 and 1995 listed in Table 2 (hereafter denoted observational catalogues). It gives, on average, 20 observations per star spread over 40 years. This means that we can investigate the non-linear motions of stars with periods from about 10 to 100 years and amplitudes >100 mas. Apparently, the use of a few accurate catalogues observed before 1939 would add little to the investigation of non-linear motions of bright stars, but they may be appropriate for investigating fainter stars with poor observational histories.

\section{Method}

We used the positions and parallaxes from the HIP; radial velocities from the HIPPARCOS input catalogue (HIC) (Turon et al. 1993); the proper motions from the HIP and best ground-based compilations: GC (Boss 1937), N30 (Morgan 1952), FK5, N70E (Kolesnik 1997), CMC9 (Carlsberg Meridian Catalogue \# 9 1997), KSV2 (Time Service Catalogue 2) (Gorshkov \& Scherbakova 1998); and the positions from the observational catalogues. All mentioned ground-based catalogues were transferred to the equinox J2000.

Since the discrepancies between HIP and ground-based proper motions of non-linearly moving stars may be great (sometimes >50 mas/year), proper motions from the HIP, GC, N30, FK5, N70E, CMC9 and KSV2 were used for the calculation of mean weighted proper motions as initial values for the main procedure. These ground-based compilation catalogues were first adjusted to the HIPPARCOS system: the proper motion differences in the sense "catalogue minus HIP" were approximated by polynomials, which were then eliminated from the ground-based proper motions. The weights were calculated from an intercomparison of the catalogues by the method described by Zverev et al. (1980). We did not need a more rigorous reduction of these catalogues because these mean proper motions were used only as initial values for the first of three iterations. The proper motions from these catalogues have no influence on the final result.

The main procedure was:

1. All stars were divided into linearly moving ones (mainly single stars, classes "S" and "W" discussed later), non-linearly moving ones (class "A" discussed later) and stars with doubtful results (class "C" discussed later). Among the non-linearly moving stars are those with calculated proper motion components differing from the HIP by $>7$ mas/year $(\Delta \mu$ binaries in terms of the FK6). Thus, the lists of linearly and non-linearly moving stars were revised as the proper motions were recalculated in every iteration;

2. The calculated proper motions together with positions and parallaxes from the HIP and radial velocities from the HIC were used to calculate the positions for the epochs of the observational catalogues following the procedure described in (ESA 1997, vol. 1, Sect. 1.5.5);

3. The differences $\Delta \alpha$ and $\Delta \delta$ in the sense "observational catalogue minus HIP at the observational catalogue epoch" for every linearly moving star in every observational catalogue were calculated;

4. The systematic behaviour in these differences was approximated by Legendre-Hermite-Fourier functions of $\alpha$ and $\delta$. After elimination of the systematic curve the autocorrelation function of the residuals on the sphere was calculated as described by Gubanov \& Titov (1993). The $\gamma$-criterion was used to test the separation of the systematic and accidental components as described by Zverev et al. (1980);

5 . The approximating functions were eliminated from the coordinates of all stars in the observational catalogues. Thus, the observational catalogues were reduced to the HIPPARCOS (ICRS) system with mean errors in the range 30-200 mas, varying from catalogue to catalogue as tested by the intercomparison of the catalogues with close epochs. The weights of the catalogues were calculated from their mean errors. It turns out from the comparison of these weights that three long series of observations made with the Washington 6-inch transit circle, Hamburg meridian telescope at Perth and Carlsberg meridian circle at La Palma are of exceptional importance in our investigation;

6 . The mean weighted proper motions were recalculated using the series of the reduced ground-based positions together with the HIPPARCOS position for 1991.25;

7. These proper motions were then used for the next two iterations (steps 1-6) until the obtained improvements were insignificant.

The advantage of the obtained PMFS proper motions over ground-based ones (such as from the FK5) is that the PMFS proper motion system is very close to the HIP one. 
It shows itself in the fact that the differences "PMFS minus HIP" do not indicate noticeable systematic dependence on the equatorial coordinates. On the other hand, the advantage of the PMFS proper motions over HIP ones is that they reflect the star motions over decades, not only in the course of 3.36 years of the mission. Namely, the nonlinear motions of 134 stars were directly separated into their proper motions and periodical motions of the photocentres around barycentres, and the proper motions of some other 200 stars were separated from their non-linear motions which were implicit in large differences between the PMFS and HIP proper motions of the stars.

\section{Stellar systems}

There are many double and multiple stellar systems in the Basic FK5. The stars are divided into 4 classes. 760 stars can be considered as single. They are marked as the class "S". All the pairs with separation $>10$ arcsec and $>10000$ AU are likely to be common proper motion stars or optical pairs. Their bright components are also marked as single stars.

In the case of wide pairs (separation $>10$ arcsec but $<10000 \mathrm{AU}$, no orbital motion) the fainter component usually does not affect the ground-based or space astrometry of the brighter one. In this case the method used and the obtained proper motions are acceptable. $187 \mathrm{such}$ stars are marked as the class "W". Closer pairs need some special consideration.

The parameters (resolving power, field of view etc.) of the astrometric telescopes are so different that whereas one of them may resolve a pair and observes the brighter component, another resolves the pair and observes its photocentre, while yet another does not resolve the pair and observes its photocentre. Therefore, any combination of the results of different telescopes (in our study also) may be doubtful for some pairs, mainly with the separation from 1 to 10 arcsec. 35 such stars are marked as class "C".

Some of the stars have been known as spectroscopic, eclipsing, close visual binaries or even single ones, but in the series of astrometric observations with different epochs they appear as non-linearly moving photocentres of astrometrically unresolved stellar systems with some hidden (i.e. faint) massive components. Such stellar systems are known as astrometric binaries (see ESA 1997). The class "A" (astrometric binaries) in the PMFS includes 551 stellar systems with 553 brighter components in the Basic FK5 for which the astrometrically observed photocentre moves, or can move, non-linearly with an amplitude $>1$ mas:

1. the visual or photocentre orbit is well-known (in fact it means a period $\ll 1000$ years), or

2 . suspected revolution period is $<200$ years, or

3. the distance between components is $<75$ AU (one suspect a rather short period in this case), or
Table 1. The format of the catalogue of proper motions of fundamental stars

\begin{tabular}{rlll}
\hline Bytes & Format & Units & Explanations \\
\hline $1-6$ & I6 & & {$[122,118322]$ HIPPARCOS number } \\
$7-11$ & I5 & & {$[1,1670]$ FK5 number } \\
$12-19$ & F8.1 & mas/year & {$[-3629.0,4141.8] \mu_{\alpha} \cos \delta$} \\
$20-23$ & F4.1 & mas/year & {$[0.4,2.0]$ Mean error of $\mu_{\alpha} \cos \delta$} \\
$24-31$ & F8.1 & mas/year & {$[-5811.1,3267.5] \mu_{\delta}$} \\
$32-35$ & F4.1 & mas/year & {$[0.3,2.0]$ Mean error of $\mu_{\delta}$} \\
$36-36$ & I1 & blank \\
$37-37$ & A1 & [ACSW] Class of stellar system \\
\hline
\end{tabular}

4. a spectroscopic or eclipsing component is known and we suspect the photocentre periodic motion amplitude $>1$ mas, or

5. the distance between components is $<1$ arcsec (in this case many astrometric telescopes did not resolve the system and observe its photocentre), or

6. the HIP Double/Multiple System flag (field H59) is O, $\mathrm{G}, \mathrm{V}$ or $\mathrm{X}$, or

7. the HIP solution quality (field H61) is S, or

8. the absolute difference between the HIP and PMFS proper motions is $>7$ mas/year for either $\mu_{\alpha} \cos \delta$ or $\mu_{\delta}$, or

9. we found a non-linear motion of the photocentre.

One would expect a significant diversity of the proper motions of these stars and stellar systems in different catalogues. The PMFS proper motions are the linear motions of the barycentres of these systems. Some parameters of a periodic motion of their photocentres around barycentres have been obtained also and combined with the parameters known from other sources in a separate catalogue of astrometric binaries, which will be published elsewhere.

\section{Statistics and format of the catalogue}

The PMFS catalogue gives the proper motion components $\mu_{\alpha} \cos \delta$ and $\mu_{\delta}$ in mas/year, their mean errors and classification of the stellar systems by the type of duplicity. The PMFS is presented in Table 3 deposited in machine-readable form at the CDS. The mean error of the proper motion components varies from 0.3 to $2.0 \mathrm{mas} /$ year. The median precision is $0.5 \mathrm{mas} / \mathrm{year}$ for $\mu_{\alpha} \cos \delta$ and 0.7 mas/year for $\mu_{\delta}$.

The format of the catalogue is given in Table 1 . The stars are listed in order of HIP number.

\section{Comparison with the FK6}

The proper motions of the fundamental stars in the PMFS and FK6 are quite independent. Therefore, it will be very interesting to make a detailed comparison of these catalogues when all parts of the FK6 have been completed. At present we restrict our comparison to the proper motions 
Table 2. The observational ground-based catalogues used for star positions

\begin{tabular}{|c|c|c|c|c|c|}
\hline Abbreviation & Name in CDS & Observatory & Telescope & Reference & Epoch \\
\hline W150 & $\mathrm{I} / 100 \mathrm{~A}$ & Washington & 6-inch transit circle & Watts \& Adams (1949) & 1939 \\
\hline W250 & $\mathrm{I} / 100 \mathrm{~A}$ & Washington & 6-inch transit circle & Watts et al. (1952) & 1945 \\
\hline W350 & $\mathrm{I} / 100 \mathrm{~A}$ & Washington & 6-inch transit circle & Adams et al. (1964) & 1953 \\
\hline W450 & $\mathrm{I} / 100 \mathrm{~A}$ & Washington & 6-inch transit circle & Adams et al. (1968) & 1960 \\
\hline W550 & $\mathrm{I} / 100 \mathrm{~A}$ & Washington & 6-inch transit circle & Hughes \& Scott (1982) & 1967 \\
\hline W1j00 & & Washington & 6-inch transit circle & Holdenried \& Rafferty (1997) & 1980 \\
\hline WL50 & & El Leoncito & 7-inch transit circle & Hughes et al. (1992) & 1971 \\
\hline PU55A & & Pulkovo & Tepfer meridian circle & Bedin et al. (1983) & 1955 \\
\hline PU58A & & Pulkovo & Pulkovo large transit circle & Nemiro et al. (1977) & 1958 \\
\hline PU58D & & Pulkovo & Ertel-Struve vertical circle & Bagildinsky \& Kossin (1966) & 1958 \\
\hline PU72D & & Pulkovo & Ertel-Struve vertical circle & Bagildinsky et al. (1986) & 1972 \\
\hline SPU66D & & Cerro Calan & photographic vertical circle & Naumov (2000) & 1966 \\
\hline PVC96 & $\mathrm{I} / 242$ & Pulkovo & photographic vertical circle & Bagildinsky et al. (1998) & 1991 \\
\hline SPF1A & $\mathrm{I} / 82$ & Cerro Calan & Repsold meridian circle & Anguita et al. (1975) & 1965 \\
\hline SPF1DS & $\mathrm{J} / \mathrm{A}+\mathrm{AS} / 67 / 1$ & Cerro Calan & Repsold meridian circle & Carrasco \& Loyola (1987) & 1965 \\
\hline SPF1DP & & Cerro Calan & Repsold meridian circle & Gnevysheva \& Zverev (1983) & 1965 \\
\hline SPF2 & $\mathrm{I} / 44$ & Cerro Calan & Repsold meridian circle & Baturina et al. (1986) & 1966 \\
\hline SPF3 & $\mathrm{I} / 78$ & Cerro Calan & Zeiss transit instrument & Loyola \& Shishkina (1974) & 1964 \\
\hline Santiago67 & $\mathrm{I} / 89 \mathrm{~A}$ & Cerro Calan & Repsold meridian circle & Carrasco \& Loyola (1981) & 1967 \\
\hline SantiagoFC83 & $\mathrm{J} / \mathrm{A}+\mathrm{AS} / 95 / 355$ & Cerro Calan & Repsold meridian circle & Carrasco \& Loyola (1992) & 1983 \\
\hline SPU71A & & Cerro Calan & Pulkovo large transit circle & Varin et al. (1981) & 1971 \\
\hline Nikolaev60A & & Nikolaev & transit circle & Markina \& Petrov (1969) & 1960 \\
\hline Nikolaev60D & & Nikolaev & vertical circle & Bozhko \& Zimmermann (1977) & 1960 \\
\hline Goloseevo81D & & Goloseevo & vertical circle & Lazorenko (1985) & 1981 \\
\hline Brorfelde68 & $\mathrm{I} / 30$ & Brorfelde & Carlsberg meridian circle & Olsen Fogh et al. (1973) & 1968 \\
\hline Brorfelde73 & $\mathrm{I} / 99$ & Brorfelde & Carlsberg meridian circle & Helmer \& Olsen Fogh (1982) & 1973 \\
\hline Brorfelde82 & $\mathrm{I} / 99$ & Brorfelde & Carlsberg meridian circle & Helmer et al. $(1983,1984)$ & 1982 \\
\hline CMC1 & $\mathrm{I} / 126$ & La Palma & Carlsberg meridian circle & CMC (1989) & 1984 \\
\hline $\mathrm{CMC} 2$ & $\mathrm{I} / 126$ & La Palma & Carlsberg meridian circle & CMC (1989) & 1985 \\
\hline CMC3 & $\mathrm{I} / 133$ & La Palma & Carlsberg meridian circle & CMC (1989) & 1986 \\
\hline CMC4 & $\mathrm{I} / 147$ & La Palma & Carlsberg meridian circle & CMC (1989) & 1987 \\
\hline CMC5 & $\mathrm{I} / 170 \mathrm{~A}$ & La Palma & Carlsberg meridian circle & CMC (1991) & 1989 \\
\hline CMC6 & $\mathrm{I} / 189$ & La Palma & Carlsberg meridian circle & CMC (1992) & 1990 \\
\hline $\mathrm{CMC} 7$ & $\mathrm{I} / 205$ & La Palma & Carlsberg meridian circle & CMC (1993) & 1991 \\
\hline CMC8 & $\mathrm{I} / 213$ & La Palma & Carlsberg meridian circle & CMC (1994) & 1993 \\
\hline CMC9 & & La Palma & Carlsberg meridian circle & CMC (1997) & 1994 \\
\hline Perth70 & $\mathrm{I} / 62 \mathrm{C}$ & Perth & Hamburg meridian teles. & Høg \& von der Heide (1976) & 1970 \\
\hline Perth75 & $\mathrm{I} / 97$ & Perth & Hamburg meridian teles. & Nikoloff \& Høg (1991) & 1975 \\
\hline Perth83 & $\mathrm{I} / 168$ & Perth & Hamburg meridian teles. & Harwood (1990) & 1984 \\
\hline $\mathrm{BMC} 74$ & $\mathrm{I} / 63$ & Bordeaux & meridian circle & Mazurier et al. (1977) & 1974 \\
\hline BMC89 & & Bordeaux & meridian circle & Requieme (1993) & 1989 \\
\hline Beograd69D & & Beograd & vertical circle & Sadzakov \& Saletic (1972) & 1969 \\
\hline Beograd84 & $\mathrm{J} / \mathrm{A}+\mathrm{AS} / 77 / 411$ & Beograd & meridian circle & Sadzakov \& Dacic (1989) & 1984 \\
\hline CPASJ2 & $\mathrm{J} / \mathrm{A}+\mathrm{AS} / 136 / 1$ & San Juan & Beijing astrolabe & Manrique et al. (1999) & 1994 \\
\hline PACP4 & $\mathrm{I} / 182$ & Beijing & photoelectric astrolabe & Lu Lizhi (1991) & 1987 \\
\hline PPCP3 & & Beijing & transit instrument & Lu Lizhi (1990) & 1982 \\
\hline PPCP4 & & Beijing & transit instrument & Wang \& Lu Lizhi (1991) & 1990 \\
\hline QAC2 & $\mathrm{J} / \mathrm{A}+\mathrm{AS} / 103 / 427$ & Quito & Danjon astrolabe OPL-13 & Kolesnik \& Davila (1994) & 1970 \\
\hline SAC1 & $\mathrm{J} / \mathrm{A}+\mathrm{AS} / 18 / 135$ & Cerro Calan & Santiago Danjon astrolabe & Noël et al. (1974) & 1969 \\
\hline $\mathrm{SAC} 2$ & $\mathrm{~J} / \mathrm{A}+\mathrm{AS} / 106 / 441$ & Cerro Calan & Santiago Danjon astrolabe & Noël (1994) & 1980 \\
\hline CERGA92 & $\mathrm{J} / \mathrm{A}+\mathrm{AS} / 96 / 477$ & CERGA & photoelectric astrolabe & Vigouroux et al. (1992) & 1990 \\
\hline Calern99 & $\mathrm{J} / \mathrm{A}+\mathrm{AS} / 137 / 269$ & Calern & photoelectric astrolabe & Martin et al. (1999) & 1995 \\
\hline GCA & $\mathrm{J} / \mathrm{A}+\mathrm{AS} / 31 / 159$ & various & various astrolabes & Billaud et al. (1978) & 1967 \\
\hline KSV (TSC) & & various & various & Pavlov (1971) & 1958 \\
\hline KSV2 (TSC2) & & various & various & Gorshkov \& Scherbakova (1998) & 1982 \\
\hline GCPA & $\mathrm{I} / 180$ & various & various astrolabes & GCPA (1992) & 1979 \\
\hline GCPA2 & $\mathrm{I} / 181$ & various & various astrolabes & Lu Lizhi (1992) & 1987 \\
\hline
\end{tabular}




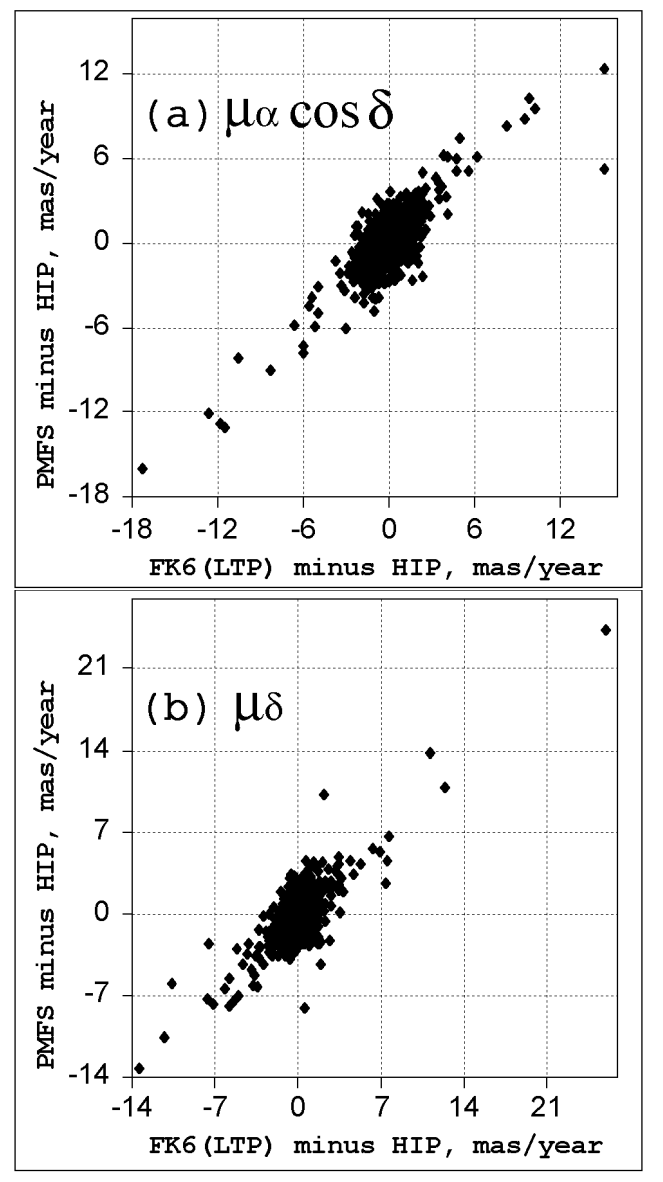

Fig. 1. The proper motion differences in mas/year in the sense "FK6 minus HIP" versus "PMFS minus HIP" for 878 stars from the FK6, Part I: $\left.\mu_{\alpha} \cos \delta \mathbf{a}\right), \mu_{\delta} \mathbf{b}$ )

in HIP, FK6 and PMFS of the 878 stars in Part I of the FK6.

The PMFS proper motions are derived from over 40 years of astrometric observations and can be considered as a "long-term" prediction of coordinate variations. Therefore, as recommended by Wielen et al. (1999) in Sect. 8 of the FK6, the PMFS proper motions should be compared with the long-term prediction mode of the FK6 (hereafter FK6(LTP)).

The median precision of the FK6(LTP) and PMFS is: 0.46 versus $0.50 \mathrm{mas} /$ year for $\mu_{\alpha} \cos \delta$ and 0.45 versus 0.60 mas/year for $\mu_{\delta}$. The standard deviation of the differences "FK6(LTP) minus HIP" and "PMFS minus HIP" is: 1.9 versus 2.1 mas/year for $\mu_{\alpha} \cos \delta$ and 1.9 versus $2.2 \mathrm{mas} /$ year for $\mu_{\delta}$. Thus, formally the PMFS is only about $15 \%$ less accurate than the FK6. One should expect such a situation for these linearly moving stars because the ground-based data used in the FK5 cover a longer period. However, the inverse error budget may occur for non-linearly moving stars in the other parts of the FK6.

The standard deviation of the difference "PMFS minus FK6(LTP)" is 1.3 mas/year for $\mu_{\alpha} \cos \delta$ and 1.5 mas/year for $\mu_{\delta}$. Thus, the PMFS and FK6 are closer to one another than to the HIP. A strong correlation of the differences
"FK6 minus HIP" and "PMFS minus HIP" for individual stars is evident from Fig. 1.

We conclude that, although the accuracy of the PMFS and FK6 seems overestimated, the proper motions in both catalogues compare favourably with the HIP.

Acknowledgements. We thank Dr. Leslie Morrison for improvement of the language and Prof. Roland Wielen for useful discussion.

The creators and providers of all mentioned catalogues are gratefully acknowledged. This research has made use of the Centre de Données astronomiques de Strasbourg (CDS), Astronomical Data Center (ADC) at NASA Goddard Space Flight Center and SIMBAD astronomical database.

This work was supported by the Russian foundation for basic research, RFBR, project \#97-02-17111.

\section{References}

Adams, A. N., Bestul, S. M., \& Scott, D. K. 1964, Publ. USNO, 2, 19, Part I, 111

Adams, A. N., Bestul, S. M., \& Scott, D. K. 1964, Publ. USNO, 2, 19, Part II, 295

Andronova, A. 1998, in Journées 1998, systèmes de référence spatio-temporels, ed. N. Capitaine (Édition Observatoire de Paris), 57

Anguita, C., Carrasco, G., Loyola, P., et al. 1975, Publ. Depart. Astron. Univer. Chile, 2, 181

Bagildinsky, B. K., \& Kossin, G. S. 1966, Publ. Pulkovo Obs. (Trudy GAO AN SSSR), 76, 5

Bagildinsky, B. K., Kossin, G. S., Medvedeva, L. I., et al. 1986, Publ. Pulkovo Obs. (Trudy GAO AN SSSR), 86, 159

Bagildinsky, B. K., Gontcharov, G. A., Kornilov, E. V., et al. 1998, Publ. Pulkovo Obs. (Izvestia glavnoy... ), 213, 48

Baturina, G. D., Bedin, V. S., Gnevysheva, K. G., et al. 1986, Publ. Pulkovo Obs. (Trudy GAO AN SSSR), 86, 4

Bedin, V. S., Zverev, M. S., \& Izvekova, A. A. 1983, Publ. Pulkovo Obs. (Trudy GAO AN SSSR), 85, 149

Billaud, G., Guilliano, G., \& Vigouroux, G. 1978, A\&AS, 31, 159

Boss, B. 1937, Publ. Carnegie Inst. of Washington, 468

Bozhko, I. I., \& Zimmermann, G. K. 1977, Publ. Pulkovo Obs. (Trudy GAO AN SSSR), 82, 53

Carlsberg Meridian Catalogue La Palma \# 4 1989, Copenhagen Univ. Obs., Royal Greenwich Obs., Real Inst. y Obs. de la Armada en San Fernando

Carlsberg Meridian Catalogue La Palma \# 5 1991, Copenhagen Univ. Obs., Royal Greenwich Obs., Real Inst. y Obs. de la Armada en San Fernando

Carlsberg Meridian Catalogue La Palma \# 6 1992, Copenhagen Univ. Obs., Royal Greenwich Obs., Real Inst. y Obs. de la Armada en San Fernando

Carlsberg Meridian Catalogue La Palma \# 7 1993, Copenhagen Univ. Obs., Royal Greenwich Obs., Real Inst. y Obs. de la Armada en San Fernando

Carlsberg Meridian Catalogue La Palma \# 8 1994, Copenhagen Univ. Obs., Royal Greenwich Obs., Real Inst. y Obs. de la Armada en San Fernando

Carlsberg Meridian Catalogue La Palma \# 9 1997, Copenhagen Univ. Obs., Royal Greenwich Obs., Real Inst. y Obs. de la Armada en San Fernando

Carrasco, G., \& Loyola, P. 1981, Publ. Depart. Astron. Univer. Chile, IV 
Carrasco, G., \& Loyola, P. 1987, A\&AS, 67, 1

Carrasco, G., \& Loyola, P. 1992, A\&AS, 95, 355

ESA 1997, The Hipparcos Catalogue, ESA SP-1200

Fricke, W., Schwan, H., Lederle, T., et al. 1988, Veröff. Astron. Rechen-Institut Heidelberg, 32

Fricke, W., Schwan, H., Corbin, T., et al. 1991, Veröff. Astron. Rechen-Institut Heidelberg, 33

General catalogue of stars with the system of photoelectric astrolabes 1992, Beijing, Yunnan, Shanghai and Shaanxi Astron. Obs.

Gnevysheva, K. G., \& Zverev, M. S. 1983, Publ. Pulkovo Obs. (Trudy GAO AN SSSR), 85, 197

Gontcharov, G., \& Andronova, A. 1997, in Journées 1997, systèmes de référence spatio-temporels, ed. J. Vondrak, \& N. Capitaine (Édition Observatoire de Paris and Astronomical Institute in Prague), 122

Gontcharov, G., \& Kornilov, E. 1997, in Journées 1997, systèmes de référence spatio-temporels, ed. J. Vondrak, \& N. Capitaine (Édition Observatoire de Paris and Astronomical Institute in Prague), 134

Gontcharov, G. A., Andronova, A. A., \& Kornilov, E. V. 1998, Publ. Pulkovo Obs. (Izvestia glavnoy...), 213, 8

Gorshkov, V. L., \& Scherbakova, N. V. 1998, Publ. Pulkovo Obs. (Izvestia glavnoy... ), 213, 25

Gubanov, V. S., \& Titov, O. A. 1993, Kinematika i fisika nebesnyh tel (Russian), 9, 56

Harwood, D. N. 1990, Perth83 catalogue of 12263 stars, Perth Obs.

Helmer, L., \& Olsen Fogh, H. J. 1982, A\&AS, 49, 13

Helmer, L., Fabricius, C., Einicke, O.H., et al., 1983, A\&AS 53, 223

Helmer, L., Fabricius, C., Einicke, O. H., et al. 1984, A\&AS, 55,87

Høg, E., \& von der Heide, J. 1976, Abh. der Hamburger Sternwarte, Band IX, 15

Holdenried, E., \& Rafferty, T. J. 1997, URL: http://aries.usno.navy.mil/ad/w1j00/

Hughes, J. A., \& Scott, D. K. 1982, Publ. USNO, 2, 23, Part III, 165

Hughes, J. A., Smith, C. A., \& Branham, R. L. 1992, Publ. USNO, 2, 26, Part II

Kolesnik, Y. 1997, MNRAS, 285, 1

Kolesnik, Y., \& Davila, H. 1994, A\&AS, 103, 427

Lazorenko, P. F. 1985, Publ. VINITI (Russian), \# 6260-85

Loyola, P. R., \& Shishkina, V. N. 1974, Publ. Depart. Astron. Univer. Chile, 2, 159
Lu Lizhi 1990, Publ. Beijing Astron. Obs., 17, 105

Lu Lizhi 1991, Publ. Beijing Astron. Obs., 17, 59

Lu Lizhi 1992, Bull. Inf. CDS, 40, 9

Manrique, W. T., Lu, L., Perdomo, R. et al. 1999, A\&AS, 136, 1

Markina, O. T., \& Petrov, G. M. 1969, Publ. Pulkovo Obs. (Trudy GAO AN SSSR), 77, 5

Martin, V. A. F., Leister, N. V., Vigouroux, G., et al. 1999, A\&AS, 137, 269

Mazurier, J. M., Mangenot, G., Requieme, Y., et al. 1977, A\&AS, 27, 467

Morgan, H. R. 1952, Astron. Papers Amer. Ephemeris, 13, Part III

Naumov, V. A. 2000, Publ. Pulkovo Obs. (Izvestia glavnoy...), in press

Nemiro, A. A., Pljugina, A. I., Tavastsherna, K. N., et al. 1977, Publ. Pulkovo Obs. (Trudy GAO AN SSSR), 82, 4

Nikoloff, I., \& Høg, E. 1991, Perth75: a catalogue of positions of 2589 FK4 and FK4S stars (NASA, GSFC, Greenbelt)

Noël, F. 1994, A\&AS, 106, 441

Noël, F., Czuia, K., Guerra, P., et al. 1974, A\&AS, 18, 135

Olsen Fogh, H. J., Jensen, P., \& Knudsen, T. 1973, A\&AS, 9, 1

Pavlov, N. N. 1971, Publ. Pulkovo Obs. (Trudy GAO AN SSSR), 78, 59

Réquième, Y. 1993, private communication

Sadzakov, S., \& Saletic, D. 1972, Publ. Obs. Astron. Beograd

Sadzakov, S., \& Dacic, M. 1989, A\&AS, 77, 411

Turon, C., Crézé, M., Egret, D., et al. 1993, Version 2 of the Hipparcos Input Catalogue, tape version, Bull. Inf. CDS, 43,5

Varin, M. P., Varina, V. A., Gubanov, V. S., et al. 1981, Publ. Pulkovo Obs. (Trudy GAO AN SSSR), 84, 4

Vigouroux, G., Billaud, G., Boche, R., et al. 1992, A\&AS, 96, 477

Wang, R., \& Lu, L. 1991, Publ. Beijing Astron. Obs., 17, 120

Watts, C. B., \& Adams, A. N. 1949, Publ. USNO, 2, 16, Part I, 255

Watts, C. B., Scott, F. P., \& Adams, A. N. 1952, Publ. USNO, 2, 16, Part III, 447

Wielen, R., Schwan, H., Dettbarn, C., et al. 1999, Veröff. Astron. Rechen-Institut Heidelberg, 35

Zverev, M. S., Kuryanova, A. N., Polojentsev, D. D., et al. 1980, Compiled catalogue of the fundamental faint stars with the declinations from $+90^{\circ}$ to $-20^{\circ}$, Kiev, Naukova dumka 